\title{
EFFECTS OF INDIRECT NUTRITIONAL INTERVENTION BY A REGISTERED DIETITIAN THROUGH VISITING NURSES IN NUTRITIONALLY AT-RISK OLDER HOME-CARE RECIPIENTS: A RANDOMIZED PILOT STUDY
}

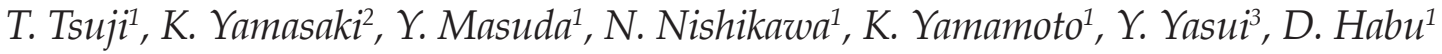

\begin{abstract}
Objectives: Home-visit nutritional support services by registered dietitians for home-care patients are not popular due to a shortage of visiting registered dietitians. This study examined the effects of indirect nutritional intervention by a registered dietitian through visiting nurses in community-dwelling older home-care recipients. Design: A randomized, open-label, controlled clinical trial. Setting: Community. Participants: Thirty-four nutritionally at-risk older home-care recipients aged 65 years or older who were receiving home-visit nursing care services. Intervention: Dietary counseling promoting highly varied food intake provided by visiting nurses trained by a registered dietitian. Measurements: Nutritional status based on the Mini Nutritional Assessment-Short Form (MNA®-SF), dietary variety, anthropometric measurements, activities of daily living, and subjective diet-related quality of life were measured at baseline and after three months. Results: Nutritional status tended to improve in the intervention group $(\mathrm{P}=0.087)$. Analysis of the changes of MNA®-SF item scores revealed significant improvement in "Appetite loss" $(\mathrm{P}=0.046)$ and a trend toward improvement in "Weight loss" $(\mathrm{P}=0.053)$ in the intervention group. Conclusion: Indirect nutritional intervention by a registered dietitian through visiting nurses was effective in improving the nutritional status of nutritionally at-risk older home-care recipients.
\end{abstract}

Key words: Malnutrition, older home-care recipients, dietary variety, registered dietitian, visiting nurse.

\section{Introduction}

With improved life expectancy among the elderly, increased health care spending and care methods have become issues in many developed countries. Preventing care needs level becoming more severe and prolonging home care may be effective methods for reducing health care costs (1). On the other hand, many people prefer to receive home care and to die at home $(2,3)$. Japan is aging at a pace unparalleled in other countries; a survey of the elderly in Japan found that around $40 \%$ of individuals prefer to receive home care and more than half prefer to die at home (4). For these reasons, Japan is promoting home care and the number of home-care patients has been steadily increasing.

However, malnutrition is prevalent among

1. Department of Medical Nutrition, Graduate School of Human Life Science, Osaka City University, Japan; 2. Division of Visiting Nursing, Nishinomiya Social Welfare Corporation, Japan; 3. Department of Clinical Nutrition, Graduate School of Human Life Science, Osaka City University, Japan.

Corresponding Author: Taeko Tsuji, Department of Medical Nutrition, Graduate School of Human Life Science, Osaka City University, 3-3-138 Sugimoto, Sumiyoshi-ku, Osaka city, Osaka, 558-8585, Japan. E-mail: taeko.t1110@gmail.com TEL: +81-(0)6-6605-2892 FAX: +81-(0)6-6605-2892

Received September 13, 2016

Accepted for publication January 3, 2017 community-dwelling older individuals, especially in home-care recipients $(5,6)$. Malnutrition causes adverse events such as increased infection rates, delayed wound healing, and decreased longevity $(7,8)$. Additionally, malnourished elderly are more likely to require hospital or nursing home admission $(9,10)$. Therefore, it is important to evaluate the nutritional risk of older homecare recipients early in order to prevent malnutrition and improve nutritional status so that the elderly can continue home care.

Japan offers home-visit nutritional support for homecare patients from registered dietitians. This service has been shown to reduce malnutrition and improve blood glucose level, activity of daily living (ADL), and quality of life (QOL) (11, 12). However, there remain many unserved cases and a burgeoning need for home-visit nutritional support due to a shortage of visiting registered dietitians $(13,14)$.

In order to address this problem, we focused on utilizing visiting nurses as human medical resources. Nurses are specialists who support patients by providing medical treatment and nutritional management as a part of their nursing role. In addition, visiting nurses play an 
essential role in malnutrition prevention and treatment (15). However, their lack of knowledge about nutrition and nutritional counseling has been pointed out in practice $(15,16)$. Furthermore, a previous study reported that visiting nurses underestimate the prevalence of malnutrition in their patients (15).

Therefore, we hypothesized that cooperation and complementation between registered dieticians and visiting nurses might prevent malnutrition in older homecare recipients. We developed an indirect nutritional intervention by a registered dietitian through visiting nurses. In this strategy, a registered dietitian educated visiting nurses about dietary counseling aimed at improving older home-care recipients' nutritional status; the trained nurses then provided dietary counseling to their patients.

The characteristics of nutritionally at-risk homecare patients were as follows. Although it was difficult to determine at a glance that they were at risk of malnutrition, the dietary variety in this at-risk population tended to be lower than that of well-nourished patients (17). On the other hand, highly varied diets have been associated with better nutritional status (18). In previous studies promoting varied food intake in functionally competent elderly, dietary variety and nutritional status were improved $(19,20)$. In addition, because dietary variety has the advantages of being teachable and easy to understand, it has been utilized as a simple and valuable approach for nutritional interventions in the elderly (19-21). Consequently, dietary counseling using dietary variety as an intervention point is suitable for indirect intervention by a registered dietitian.

The purpose of this study was to examine the effects of a preventive measure against malnutrition for nutritionally at-risk older home-care recipients by an indirect nutritional intervention by a registered dietitian through visiting nurses.

\section{Materials and Methods}

\section{Study design and subjects}

This study was a randomized, open-label, controlled clinical trial performed in a single city, Hyogo Prefecture, Japan between July 2014 and February 2015. Communitydwelling older care recipients (aged 65 years or older who were receiving home-visit nursing care services), who were at risk of malnutrition according to the Mini Nutritional Assessment-Short Form (MNA®-SF) (22-25), were included in this study. Subjects were excluded from the study if they had a medical condition preventing oral feeding, were undergoing dietary therapy, were suffering from severe dysphagia or kidney or liver diseases, were end-of-life patients, or were unable to or willing to provide informed consent. Thirty-four older care recipients were included in this study and alternately randomized into either the control $(n=15)$ or intervention $(n=19)$ groups. Through ethical considerations, all participants were provided a leaflet with general information about healthy eating habits for the elderly.

\section{Intervention}

Participants allocated to the control group received usual nursing care, while participants and/or their caregivers allocated to the intervention group received dietary counseling that promoted highly varied food intake using an educational leaflet provided by a visiting nurse. Visiting nurses received nutritional training from a registered dietitian. The educational leaflet used for dietary counseling described foods and menus in the 10 food groups based on the dietary variety score (26) described below. The visiting nurses performed follow-up activities on each visit for three months after providing dietary counseling.

\section{Outcome measures}

A follow-up examination was conducted three months after the baseline examination. All examinations were conducted by visiting nurses at the participants' homes. Socio-demographic factors were assessed at the baseline examination. Other measures were assessed at both the baseline and follow-up surveys.

The primary outcome of this study was nutritional status and the secondary outcomes included dietary variety, anthropometric measurements, activities of daily living (ADL), and subjective diet-related quality of life (SDQOL).

\section{Nutritional assessment}

We assessed subject nutritional status using the Mini Nutritional Assessment-Short Form (MNA®-SF) (22-25). The MNA ${ }^{\circledR}-S F$ is a simple and noninvasive nutritional assessment tool for the elderly ( $\geq 65$ years). The MNA®-SF consists of six items; decreased food intake in the preceding three months, weight loss during the preceding three months, mobility, psychological stress or acute disease in the preceding three months, neuropsychological problems, and body mass index. The MNA®-SF classifies individuals as malnourished (0-7 points), at risk of malnutrition (8-11 points), and wellnourished (12-14 points).

\section{Dietary variety}

Dietary variety was evaluated using the dietary variety score (DVS) (26). The DVS is a simple dietary survey method that asks respondents how frequently they consume the 10 main food groups in Japanese meals (fish and shellfish, meat, eggs, milk, soybean products, darkcolored vegetables, seaweeds, fruits, potatoes, and oils). 
Participants respond with the following answers: "eat almost every day," "eat once every two days," "eat once or twice a week," or "seldom eat," and the total score is the sum of the scores for the 10 food groups. The original evaluation method was developed by Kumagai et al. (26) and assigned one point for the response "eat almost every day" and evaluated variety on a scale from 0 to 10 points. We considered that having a highly varied diet every day could be a burden on home-care recipients and that it was important to evaluate dietary variety including the responses "eat once every two days" and "eat once or twice a week" in order to assess the dietary habits of this population. Thus, we used the modified evaluation method developed by Fukasaku et al. (27), in which the response "eat almost every day" was scored as 4 points, "eat once every two days" as 3 points, "eat once or twice a week" as 2 points, and "seldom eat" as 1 point (score range, 10-40).

\section{Anthropometry}

Anthropometric measurements, including arm circumference (AC), triceps skinfold thickness (TSF), and calf circumference (CC), were measured using an Insertape (Abbott Japan, Tokyo, Japan), Adipometer (Abbott Japan), and MNA® CC measure (Nestle, Kobe, Japan). Arm muscle circumference (AMC) and arm muscle area (AMA) were calculated with formulas that used AC and TSF. The values of $\% \mathrm{AC}, \% \mathrm{TSF}, \% \mathrm{CC}, \% \mathrm{AMC}$, and $\%$ AMA were calculated using the medians determined by the gender and age divisions of the Japanese Anthropometric Reference Data (JARD2001) (28).

\section{$A D L$}

ADL was evaluated using the Barthel index (29) (score range, 0-100), which consists of 10 items: feeding, chair/ bed transfers, personal hygiene, toilet use, bathing, ambulation, stairs climbing, dressing, bowel control, and bladder control.

\section{$S D Q O L$}

SDQOL was evaluated using the SDQOL scale (30). The SDQOL scale is an instrument that evaluates the subjective diet-related quality of life such as diet satisfaction and enjoyment. The SDQOL scale is composed of four items: "Mealtimes are fun," "I can hardly wait for mealtime," "The atmosphere of the dining table is cheerful," "I'm satisfied with daily meals". Participants respond with a five-point scale ranging from "applicable" to "not applicable" and the total score is the sum of the four items $(1=$ not applicable to $5=$ applicable).

\section{Statistical analysis}

Outcomes are presented as the medians (25th percentile, 75th percentile) or numbers of subjects (\%). We compared the baseline characteristics between subjects in each group using Mann- Whitney U, Chi-square, or Fisher's exact tests. Wilcoxon signed rank sum tests were performed to compare each outcome before and after the intervention. IBM ${ }^{\circledR}$ SPSS ${ }^{\circledR}$ Statistics 23 software (IBM Japan, Tokyo, Japan) was used for the analysis. A p-value of less than 0.05 was considered indicative of statistical significance.

\section{Results}

\section{Participant characteristics}

The participant flow of this study is shown in Figure 1. A total of 24 participants completed the three-month examinations.

\section{Figure 1}

Study flowchart. MNA®-SF: Mini Nutritional Assessment-Short Form; DVS: Dietary variety score; AC: Arm circumference; TSF: Triceps skinfold thickness; CC: Calf circumference; ADL: Activities of daily living; SDQOL: Subjective diet-related quality of life

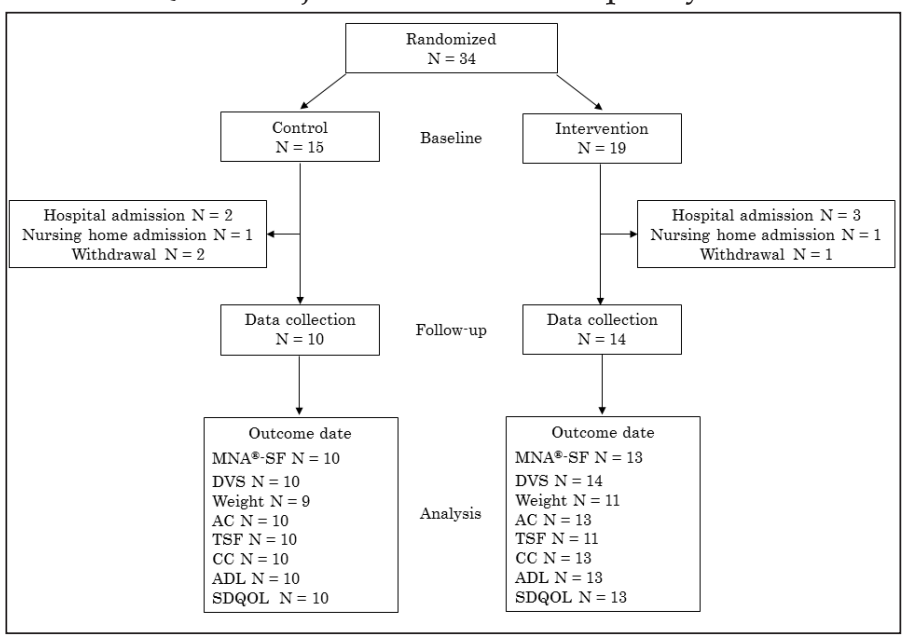

The baseline characteristics of participants in both groups are presented in Table 1 . There were no significant differences in baseline characteristics between the two groups, except for \%CC. Approximately $20 \%$ of the total study population cooked for themselves and received Meals-on-Wheels, respectively.

\section{Effects on outcomes}

Table 2 shows the changes in MNA®-SF scores, DVS, anthropometric measurements, ADL, and SDQOL in the two groups. In the intervention group, the MNA ${ }^{\circledR}-S F$ score tended to improve $(\mathrm{P}=0.087)$, although there were 
Table 1

Characteristics of each group

\begin{tabular}{|c|c|c|c|}
\hline Groups & "Control (n=15)" & "Intervention (n=19)" & P-value \\
\hline Age, years & $82.0(78.0 ; 89.0)$ & $86.0(81.0 ; 92.0)$ & .305 \\
\hline Female, $\mathrm{n}(\%)$ & $6(40.0)$ & $13(68.4)$ & .165 \\
\hline MNA®-SF, score & $9.0(8.0 ; 10.0)$ & $9.0(9.0 ; 10.0)$ & .943 \\
\hline -Appetite loss, score & $2.0(1.0 ; 2.0)$ & $2.0(1.0 ; 2.0)$ & .903 \\
\hline -Weight loss, score & $2.0(2.0 ; 3.0)$ & $2.0(2.0 ; 3.0)$ & .685 \\
\hline -Mobility, score & $1.0(1.0 ; 2.0)$ & $1.0(1.0 ; 2.0)$ & .985 \\
\hline -Psychological stress / acute disease, score & $2.0(0.0 ; 2.0)$ & $2.0(2.0 ; 2.0)$ & .227 \\
\hline -Neuropsychological disease, score & $2.0(1.0 ; 2.0)$ & $1.0(1.0 ; 2.0)$ & .137 \\
\hline -BMI/CC, score & $1.0(0.0 ; 2.0)$ & $1.0(0.0 ; 2.0)$ & .519 \\
\hline Weight, kg & $48.5(42.0 ; 56.0)$ & $46.2(36.3 ; 51.2)$ & .257 \\
\hline BMI, $\mathrm{kg} / \mathrm{m} 2$ & $21.0(16.7 ; 21.5)$ & $20.3(16.8 ; 22.4)$ & .925 \\
\hline$\% \mathrm{AC}$ & $89.7(80.4 ; 96.3)$ & $92.0(86.3 ; 108.3)$ & .306 \\
\hline$\%$ TSF & $87.5(57.1 ; 108.1)$ & $85.7(72.3 ; 122.0)$ & .899 \\
\hline$\% \mathrm{AMC}$ & $90.3(84.3 ; 97.5)$ & $94.3(90.4 ; 106.6)$ & .159 \\
\hline$\%$ AMA & $83.6(71.0 ; 95.1)$ & $90.3(81.6 ; 113.8)$ & .138 \\
\hline$\% \mathrm{CC}$ & $92.1(85.4 ; 100.0)$ & $101.4(96.3 ; 106.0)$ & .033 \\
\hline DVS, score & $31.0(29.0 ; 35.0)$ & $29.0(23.0 ; 32.0)$ & .170 \\
\hline ADL, score & $80.0(60.0 ; 95.0)$ & $80.0(75.0 ; 100.0)$ & .539 \\
\hline SDQOL, score & $13.0(12.0 ; 16.0)$ & $15.0(12.0 ; 17.0)$ & .243 \\
\hline Living alone, $\mathrm{n}(\%)$ & $3(23.1)$ & $4(21.1)$ & 1.000 \\
\hline Cooking for oneself, $\mathrm{n}(\%)$ & $5(38.5)$ & $3(16.7)$ & .228 \\
\hline Receive Meals-on-wheels, $\mathrm{n}(\%)$ & $3(23.1)$ & $3(17.6)$ & 1.000 \\
\hline
\end{tabular}

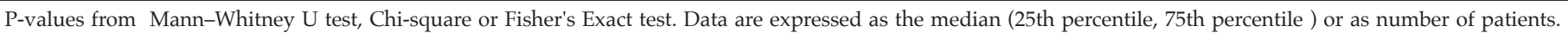

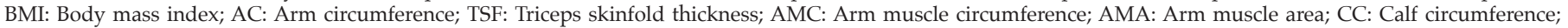
DVS: Dietary variety score; ADL: Activities of daily living; SDQOL: Subjective diet-related quality of life.

Table 2

Change in study variables after 3 months

\begin{tabular}{|c|c|c|c|c|c|c|}
\hline \multirow[t]{2}{*}{ Groups } & \multicolumn{3}{|c|}{ Control } & \multicolumn{3}{|c|}{ Intervention } \\
\hline & Baseline & After 3 months & P-value & Baseline & After 3 months & \\
\hline MNA®-SF, score & $9.0(8.8 ; 10.3)$ & $10.0(7.0 ; 11.3)$ & .677 & $9.0(8.0 ; 10.0)$ & $10.0(9.0 ; 11.0)$ & .087 \\
\hline DVS, score & $34.0(29.0 ; 36.0)$ & $33.0(29.0 ; 37.0)$ & .944 & $29.5(22.8 ; 32.8)$ & $30.5(25.3 ; 33.8)$ & .443 \\
\hline $\mathrm{BW}, \mathrm{kg}$ & $45.8(35.0 ; 56.3)$ & $46.2(35.5 ; 57.2)$ & .865 & $47.0(40.0 ; 51.2)$ & $47.6(40.0 ; 54.0)$ & .285 \\
\hline $\mathrm{BMI}, \mathrm{kg} / \mathrm{m} 2$ & $21.1(17.5 ; 21.9)$ & $20.7(18.6 ; 21.5)$ & 1.000 & $20.8(18.3 ; 22.9)$ & $21.5(18.3 ; 22.7)$ & .515 \\
\hline$\% \mathrm{AC}$ & $89.5(78.2 ; 96.5)$ & $89.7(76.8 ; 102.3)$ & .575 & $92.0(88.6 ; 101.3)$ & $95.6(87.5 ; 105.4)$ & .433 \\
\hline$\% \mathrm{TSF}$ & $88.2(54.5 ; 107.8)$ & $91.3(31.8 ; 112.8)$ & .889 & $95.0(80.0 ; 128.0)$ & $97.5(72.0 ; 124.0)$ & .678 \\
\hline$\% \mathrm{AMC}$ & $90.4(83.3 ; 98.4)$ & $90.4(82.5 ; 97.7)$ & .721 & $94.5(91.9 ; 101.4)$ & $96.0(94.8 ; 103.4)$ & .213 \\
\hline$\%$ AMA & $83.7(69.4 ; 97.0)$ & $82.8(69.3 ; 95.4)$ & .878 & $89.8(84.4 ; 102.8)$ & $92.2(90.6 ; 106.9)$ & .213 \\
\hline$\% \mathrm{CC}$ & $91.2(84.2 ; 97.9)$ & $90.9(84.2 ; 110.0)$ & .173 & $101.4(98.0 ; 103.9)$ & $100.5(96.1 ; 103.7)$ & .650 \\
\hline ADL, score & $80.0(60.0 ; 100.0)$ & $80.0(63.8 ; 100.0)$ & .330 & $80.0(70.0 ; 100.0)$ & $80.0(60.0 ; 95.0)$ & .180 \\
\hline SDQOL, score & $14.5(12.0 ; 16.3)$ & $14.5(13.0 ; 16.3)$ & .952 & $15.0(11.0 ; 17.5)$ & $16.0(12.5 ; 18.5)$ & .458 \\
\hline
\end{tabular}

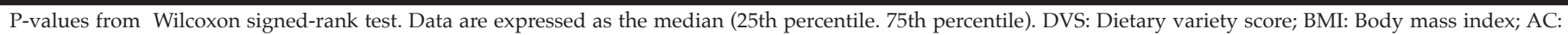

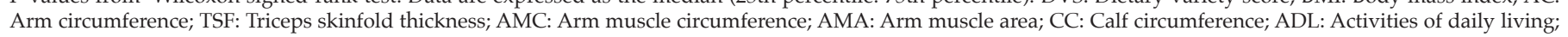
SDQOL: Subjective diet-related quality of life. 
Table 3

Changes in MNA®-SF items after 3 months

\begin{tabular}{|c|c|c|c|c|c|c|}
\hline \multirow[t]{2}{*}{ Groups } & \multicolumn{3}{|c|}{ Control $(n=10)$} & \multicolumn{3}{|c|}{ Intervention $(n=13)$} \\
\hline & Baseline & After 3 months & P-value & Baseline & After 3 months & P-value \\
\hline Appetite loss, score & $2.0(1.0 ; 2.0)$ & $2.0(1.0 ; 2.0)$ & .317 & $2.0(1.0 ; 2.0)$ & $2.0(2.0 ; 2.0)$ & .046 \\
\hline Weight loss, score & $2.0(2.0 ; 3.0)$ & $3.0(1.8 ; 3.0)$ & 679 & $2.0(2.0 ; 3.0)$ & $3.0(3.0 ; 3.0)$ & .053 \\
\hline Mobility, score & $1.0(1.0 ; 2.0)$ & $1.5(1.0 ; 2.0)$ & .564 & $1.0(1.0 ; 2.0)$ & $1.0(1.0 ; 2.0)$ & 1.000 \\
\hline Psychological stress/acute disease, score & $2.0(0.0 ; 2.0)$ & $2.0(1.5 ; 2.0)$ & .317 & $2.0(2.0 ; 2.0)$ & $2.0(0.0 ; 2.0)$ & .180 \\
\hline Neuropsychological disease, score & $2.0(1.0 ; 2.0)$ & $2.0(1.0 ; 2.0)$ & .317 & $1.0(1.0 ; 2.0)$ & $1.0(1.0 ; 2.0)$ & 1.000 \\
\hline $\mathrm{BMI} / \mathrm{CC}$, score & $1.5(0.0 ; 2.0)$ & $1.0(0.0 ; 1.3)$ & .414 & $0.0(0.0 ; 2.0)$ & $1.0(0.0 ; 2.0)$ & .180 \\
\hline
\end{tabular}

P-values from Wilcoxon signed-rank test. Data are expressed as the median (25th percentile. 75th percentile ). BMI: Body mass index; CC: Calf circumference.

no changes in DVS, anthropometric measurements, ADL, or SDQOL. In contrast, all outcome measures remained unchanged in the control group.

The changes in the scores of each MNA ${ }^{\circledR}-S F$ item are shown in Table 3. In the intervention group, there was a significant improvement in "Appetite loss" $(\mathrm{P}=0.046)$ and a trend toward improvement in "Weight loss" ( $\mathrm{P}=$ 0.053). However, there was no significant change in any of the items in the control group.

\section{Discussions}

The present study had two unique advantages. First, a registered dietitian, a specialist in nutritional management, conducted an indirect nutritional intervention through visiting nurses. These nurses received dietary counseling training from the registered dietitian aimed at improving older home-care recipients' diet and nutritional status in order to compensate for the shortage of registered dietitians providing nutritional management of home-care patients. Second, we developed a dietary counseling program using dietary variety as an intervention point as a simple, teachable and easy to understand, and valuable approach for indirect nutritional intervention by a registered dietitian.

The study intervention resulted in a trend towards improved nutritional status among nutritionally at-risk older home-care recipients. Namely, the indirect nutritional intervention by a registered dietitian through visiting nurses was effective in improving the nutritional status of the study population. In order to explore factors associated with nutritional status, we assessed the changes in score for each item on the MNA-SF®. In this study, "Appetite loss" improved significantly and "Weight loss" tended to improve. It is well known that malnutrition is caused by low and/or decreased food intake $(15,31)$. However, few home-care patients recognize the risk of appetite loss; Geurden et al. highlighted the need for educational nursing interventions (15). A possible reason for the findings of the present study is that dietary counseling in cooperation with a registered dietitian and visiting nurses improved participant food consciousness and prevented decreased food intake. Thus, participants were better able to maintain their weight. Because it is difficult for the elderly to regain food intake and weight (32), an intervention that could prevent decreased appetite and weight is of great significance.

Although we conducted dietary counseling using dietary variety as an intervention point as a simple and valuable approach in the present study, dietary counseling alone did not improve the dietary variety. Moreover, significant changes in anthropometric measurements, ADL, and SDQOL were also not observed. However, dietary variety has been associated with anthropometric measurements, ADL, and satisfaction with dietary habits $(18,33,34)$. Therefore, the lack of change in these measurements was likely due to the lack of change in dietary variety.

Previous studies on improved dietary variety included a dietary guideline aimed at improving nutritional status; the guideline had a wide range of content such as cooking method and care for dental prosthesis in addition to factors promoting highly varied food intake $(19,20)$. In addition, applicants received individualized nutritional counseling by registered dietitians (19). In the present study, for effective utilization of human medical resources, we stratified the participants according to their risks of malnutrition and visiting nurses trained by a registered dietitian conducted standardized dietary counseling for less severe nutritionally at-risk participants. Thus, we could not consider individual dietrelated living conditions such as food preferences, dietary habits, use of Meals-on-Wheels, and persons in charge of cooking. Moreover, because deficient food groups and food frequency vary in older individuals, it is necessary to set individual goals based on dietary variety and to conduct tailored dietary counseling.

Approximately $20 \%$ of the total study population in the present study cooked for themselves. In the remaining cases, family or caregivers had assumed responsibility for meal preparation. Improving dietary variety by oneself was considered to be difficult if home-care recipients gained knowledge about improving dietary habits. However, older care recipients tend not to be able to request the food they desire out of consideration 
for their caregivers (35). Additionally, Hirakawa et al. recommended providing tailored nutritional support to families with members in need of nursing care (36). Thus, it is necessary to discuss strategies to encourage caregiver cooperation with regards to cooking in order to support participant food preferences and dietary habits. Furthermore, in order to improve the quality of nutritional intervention, it is necessary to strengthen the cooperation between registered dietitians and visiting nurses and to establish a cooperative system to allow easily consultation regarding individual participants. In addition, because dietary treatment by a dietitian is more promising than when delivered by other professionals (37), direct and specific nutritional support by registered dietitians might be necessary in some preclinical cases of malnutrition.

Our study had several limitations. First, our study included the community-dwelling older care recipients living in a single city, Hyogo Prefecture, so our results may not apply to all older home-care recipients. Additionally, we could not examine the effect of factors related to dietary variety such as oral function, intellectual activity, and economic situation. On the other hand, although years of experience in nursing and comprehension of nutritional management affect the skill level of dietary counseling, we could not examine these factors in the present study. Large samples of community-dwelling older care recipients and further study on these factors are recommended.

However, to our knowledge, no other report has examined the effectiveness of improving nutritional status in nutritionally at-risk older home-care recipients by indirect nutritional intervention by a registered dietitian through visiting nurses. We believe our results to be valuable as a first step toward advocating preventive measures against malnutrition in older home-care recipients by effective use of medical resources.

\section{Conclusion}

Indirect nutritional intervention by a registered dietitian through visiting nurses improved the nutritional status of nutritionally at-risk older home-care recipients. The finding of this study indicate that nutritional management by cooperation between a registered dietitian and visiting nurses could contribute to the prevention of malnutrition and maintenance of home care for the elderly.

Acknowledgements: The authors are grateful to the participants as well as the staff of the Division of Visiting Nursing of the Nishinomiya Social Welfare Corporation.

\section{Conflicts of interest: The authors have no conflicts of interest.}

Ethical standards: This study was conducted in accordance with the Declaration of Helsinki and was approved by the ethics committee of Osaka City University.

\section{References}

1. Akiyama N, Fukuda T, Shiroiwa T, Murashima S. Investigating factors that influence health care costs for disabled elderly in Japan. Journal of Health Care and Society 2011;21(2):175-188.

2. Hays JC, Galanos AN, Palmer TA, McQuoid DR, Flint EP. Preference for place of death in a continuing care retirement community. Gerontologist 2001;41(1):123-128.

3. Yamagishi A, Morita T, Miyashita M, et al. Preferred place of care and place of death of the general public and cancer patients in Japan. Support Care Cancer 2012; 20:2575-2582.

4. Cabinet office, Existing State and Trends of Elderly People and thei Environment. Annual report on the aging society 2013. http://www8.cao. go.jp/kourei/english/annualreport/2013/pdf/1-2-2.pdf. Accessed 3 October 2016

5. Inoue K, Kato K. Usefulness of the Mini-Nutritional Assessment (MNA) to evaluate the nutritional status of Japanese frail elderly under home care. Geriatr Gerontol Int 2007;7:238-244.

6. Elia M \& Russell CA. Combating malnutrition: recommendations for action. A report from the Advisory Group on Malnutrition led by BAPEN. 2009.

7. Harris D, Haboubi N. Malnutrition screening in the elderly population. J R Soc Med 2005;98:411-414.

8. Kagansky N, Berner Y, Koren-Morag N, Perelman L, Knobler H, Levy S. Poor nutritional habits are predictors of poor outcome in very old hospitalized patients. Am J Clin Nutr 2005;82:784-791.

9. Covinsky KE, Martin GE, Beyth RJ, Justice AC, Sehgal AR, Landefeld CS. The relationship between clinical assessments of nutritional status and adverse outcomes in older hospitalized medical patients. J Am Geriatr Soc 1999;47:532-538.

10. Hallgren J, Ernsth Bravell M, Mölstad S, Östgren CJ, Midlöv P, Dahl Aslan AK. Factors associated with increased hospitalisation risk among nursing home residents in Sweden: a prospective study with a three-year follow-up. Int J Older People Nurs 2016;11:130-139.

1. Takei T, Hiramatsu K, Mori H, et al. Nutrition Programs by Visiting Dietitians on Elderly Diabetic Patients. Journal of the Japan Diabetes Society 2005;48:135-137. (in Japanese)

12. Inoue K, Nakamura I, Takahasi M, et al. Nutrition Intervention Methods Based on Home-visit Nutritional Support and Verification of the Resulting Improvement Effects. Journal of the Japan Dietetic Association 2012;8:656-664. (in Japanese)

13. Matsuzuki H. Long-term Care Insurance and Home-visit Nutritional and Dietary Guidance. The Japanese Journal of Nutrition and Dietetics 2002;60:310. (in Japanese)

14. Hirakawa Y, Masuda Y, Uemura K, Naito M, Kuzuya M, Iguchi A. Dietitians' understanding of personalized nutritional guidance--proposals to increase home visits by dietitians. Nihon Ronen Igakkai Zasshi 2003;40:509-514. (in Japanese)

15. Geurden B, Franck MPsych E, Lopez Hartmann M, Weyler J, Ysebaert D. Prevalence of 'being at risk of malnutrition' and associated factors in adult patients receiving nursing care at home in Belgium. Int J Nurs Pract 2015;21:635-644.

16. Mowe M, Bosaeus I, Rasmussen $\mathrm{HH}$, et al; Scandinavian Nutrition Group. Insufficient nutritional knowledge among health care workers? Clin Nutr 2008;27:196-202.

17. Tsuji T, Yamasaki K, Hayashi F, et al. Nutritional Assessment and Dietary Intake Status of Home Health Care Patients: A Pilot Cross-Sectional Study. J J Geronto 2015;1: 005

18. Bernstein MA, Tucker KL, Ryan ND, et al. Higher dietary variety is associated with better nutritional status in frail elderly people. J Am Diet Assoc 2002;102:1096-1104.

19. Kumagai $\mathrm{S}$, Shibata $\mathrm{H}$, Watanabe $\mathrm{S}$, et al. An intervention trial to postpone aging in competent elderly. Trial of nutritional improvement in the retirement home. Nihon Koshu Eisei Zasshi 1999;46:1003-1012. (in Japanese)

20. Kumagai $S$, Watanabe $S$, Shibata $H$, et al. An intervention study to improve the nutritional status of functionally competent community-living senior citizens. Geriatr Gerontol Int 2003;3:S21-S26.

21. Fukasaku T, Okuno J, Tomura S, et al. Intervention effects of inclusive support in an "exercise and a nutritional community-based prevention program" for pre-frail elderly individuals. Nihon Koshu Eisei Zasshi 2011;58:420-432. (in Japanese)

22. Vellas B, Villars H, Abellan G, et al. Overview of the MNA® - Its History and Challenges. J Nutr Health Aging 2006;10:456-465.

23. Rubenstein LZ, Harker JO, Salvà A, Guigoz Y, Vellas B. Screening for undernutrition in geriatric practice: developing the short-form mininutritional assessment (MNA-SF). J Gerontol A Biol Sci Med Sci 2001;56:M366-M372.

24. Guigoz Y. The Mini-Nutritional Assessment (MNA®) Review of the Literature - What does it tell us? J Nutr Health Aging 2006;10:466-487.

25. Kaiser MJ, Bauer JM, Ramsch C, et al; MNA-International Group. Validation of the Mini Nutritional Assessment Short-Form (MNA®-SF): A practical too for identification of nutritional status. J Nutr Health Aging 2009;13:782-788 
26. Kumagai S, Watanabe S, Shibata $\mathrm{H}$, et al. Effect of dietary variety on declines in high-level functional capacity in elderly people living in a community. Nihon Koshu Eisei Zasshi 2003;50:1117-1124. (in Japanese)

27. Fukasaku T, Okuno J, Yabusita N, Tanaka K, Yanagi H. The importance of a dietary variety in community-dwelling elderly individuals: attempt of new evaluation method. Jouenal of Gerontological Nursing and Caring Research 2011;1:10-19. (in Japanese)

28. Japanese Society of Nutritional Assessment. Japanese anthropometric reference data 2001(JARD 2001). Japanese Journal of Nutritional Assessment 2002;19(suppl):1-81. (in Japanese)

29. Mahoney FI, Barthel DW. FUNCTIONAL EVALUATION: THE BARTHEL INDEX. Md State Med J 1965;14:61-65

30. Ainuki T, Akamatsu R, Hayashi F, Takemi Y. The Reliability and Validity of the Subjective Diet-related Quality of Life (SDQOL) Scale among Japanese Adults. The Japanese Journal of Nutrition and Dietetics 2012;70:181-187. (in Japanese)

31. Westergren A, Unosson M, Ohlsson O, Lorefält B, Hallberg IR. Eatin difficulties, assisted eating and nutritional status in elderly ( $>$ or $=65$ years) patients in hospital rehabilitation. Int J Nurs Stud 2002;39:341-51.

32. Roberts SB, Fuss P, Heyman MB, et al. Control of food intake in older men. JAMA 1994;272:1601-1606.
33. Kimura Y, Wada T, Ishine M, et al. Food diversity is closely associated with activities of daily living, depression, and quality of life in communitydwelling elderly people. J Am Geriatr Soc 2009;57:922-924.

34. Shintaku K, Chisuwa N, Kobashi M, Tanaka K, Kimura M, Haruki T. Effects of a Collaborative Intervention with a Community Restaurant to Promote Healthy Dietary Habits among Elderly People: Formation of Subjective Wellbeing. The Japanese Journal of Nutrition and Dietetics 2013;71:145-154. (in Japanese)

35. Tanabe S, Inoue T. Qualitative analysis of current dietary behavior and associated factors in elderly individuals in need of care. Japanese Journal of Human Sciences of Health-Social Services 2014;21:45-56. (in Japanese)

36. Hirakawa Y, Enoki H, Uemura K. Proposal concerning personalized nutritional guidance. Nippon Ronen Igakkai Zasshi. Japanese Journal of Geriatrics 2010;47:334. (in Japanese)

37. Endevelt R, Lemberger J, Bregman J, et al. Intensive dietary intervention by a dietitian as a case manager among community dwelling older adults: the EDIT study. J Nutr Health Aging 2011;15:624-630. 